\title{
Highly Enantioselective Addition of Phenylacetylene to Aldehydes Catalyzed by a Camphorsulfonamide Ligand
}

\author{
Zhaoqing Xu, Chao Chen, Jiangke Xu, Mingbo Miao, Wenjin Yan, and Rui Wang*
}

Department of Biochemistry \& Molecular Biology, School of Life Science, Lanzhou University, Lanzhou 730000, and Key Laboratory of Bioorganic Chemistry and Molecular Engineering of Ministry of Education, College of Chemistry, Peking University, Beijing 100871, China.

e-mail: wangrui@1zu.edu.cn

\section{Supporting Information}

Camphorsulfonylamino 7 were synthesized according to literature procedures: a) Chittenden, R. A.; Cooper, G. H. J. Chem. Soc. C, 1970, 49; b) You, J. S.; Shao, M. Y.; Gau, H. M. Tetrahedron: Asymmetry 2001, 12, 2971. c) Shinichi Itsuno, Katsuhiro Watanabe, Takeshi Matsunomo, Shizue Kuroda, Ayako Yokoi and Ashraf El-Shehawy. J. Chem. Soc., Perkin Trans. 1999, 1, 2011-2016. It was purified by flash column chromatography (silica gel, 30\% EtOAc in hexane) and recrystallized in hexane- EtOAc.

Characterization of 7: White needles ( $42 \%$ overall yield). m.p. $142-143^{\circ} \mathrm{C}$. $[\mathrm{a}]^{19}{ }_{\mathrm{D}}=-36(\mathrm{c}=1.61, \mathrm{EtOH}),[\mathrm{a}]^{21}{ }_{\mathrm{D}}=+7\left(\mathrm{c}=1.8, \mathrm{CH}_{2} \mathrm{Cl}_{2}\right) .{ }^{1} \mathrm{H} \mathrm{NMR}\left(200 \mathrm{MHz}, \mathrm{CDCl}_{3}\right)$ : $\delta 0.75(\mathrm{~s}, 3 \mathrm{H}), \delta 0.89(\mathrm{~s}, 3 \mathrm{H}), \delta 0.91-0.95(\mathrm{~m}, 2 \mathrm{H}), \delta 1.05(\mathrm{~s}, 3 \mathrm{H}), \delta 1.38-1.49(\mathrm{~m}, 1 \mathrm{H})$, $\delta 1.57-1.67(\mathrm{~m}, \quad 2 \mathrm{H}), \quad \delta 2.44(\mathrm{~s}, \quad 3 \mathrm{H}), \quad \delta 2.02(\mathrm{Br}, \quad 1 \mathrm{H}), \quad \delta 3.21-3.28(\mathrm{t}, \quad \mathrm{J}=7 \mathrm{~Hz}, \quad 1 \mathrm{H})$, $\delta 3.55-3.58(\mathrm{~d}, \mathrm{~J}=7.6 \mathrm{~Hz}, 1 \mathrm{H}), \delta 5.26-5.29(\mathrm{~d}, \mathrm{~J}=6.2 \mathrm{~Hz}, 1 \mathrm{H}), \delta 7.29-7.33(\mathrm{~d}, \mathrm{~J}=8.2 \mathrm{~Hz}, 2 \mathrm{H})$, $\delta 7.74-7.78(\mathrm{~d}, \mathrm{~J}=8.2 \mathrm{~Hz}, 2 \mathrm{H}) .{ }^{13} \mathrm{C} \mathrm{NMR}(\mathrm{DEPT})\left(75 \mathrm{MHz}, \mathrm{CDCl}_{3}\right): \delta 143.3,136.9,48.9$, $46.9(\mathrm{C}) ; 129.7,127.2,79.0,60.1,50.6(\mathrm{CH}) ; 32.8,26.1\left(\mathrm{CH}_{2}\right) ; 21.5,21.4,20.8$, 11.2( $\left(\mathrm{CH}_{3}\right)$. IR (KBr) 3517, 3333, 3301, 3040, 2956, 2890, 1912, 1647, 1597, 1480, 1453, 1400, 1346, 1156, 1092, 1057, 941, 815, 740, 710, 664, $563 \mathrm{~cm}^{-1}$. MS (ESI) m/z: 322(M-H) ${ }^{-}$. Anal. Calcd. for $\mathrm{C}_{17} \mathrm{H}_{25} \mathrm{NO}_{3} \mathrm{~S}: \mathrm{C}, 63.16 ; \mathrm{H}, 7.74$. Found: $\mathrm{C}, 63.19 ; \mathrm{H}$, 7.76 .

General procedures for addition of phenylacethylene to aldehydes: All manipulations were carried out under argon atmosphere using dried and degassed solvent. The ligand $7(8.1 \mathrm{mg}, 0.025 \mathrm{mmol})$ and $\operatorname{Ti}\left(\mathrm{O}^{\mathrm{i}} \operatorname{Pr}\right)_{4}(27.4 \mu \mathrm{L}, 0.1 \mathrm{mmol})$ were

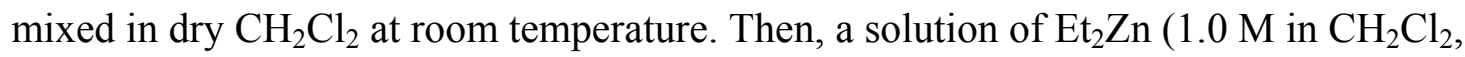


$0.75 \mathrm{~mL}$ ) was added. After the mixture was stirred at the room temperature for $2 \mathrm{~h}$, phenylacetylene $(82.4 \mu \mathrm{L}, 0.75 \mathrm{mmol})$ was added and the stirring continued for $1 \mathrm{~h}$. The yellow solution was cooled to $0^{\circ} \mathrm{C}$ and treated with aldehyde $(0.25 \mathrm{mmol})$, then the resultant mixture was allowed to warm up to room temperature and stirred for $12-14 \mathrm{~h}$. After the reaction was completed, checked by TLC, it was cooled to $0^{\circ} \mathrm{C}$ and quenched by $5 \%$ aqueous $\mathrm{HCl}$. The mixture was extracted with ether. The organic layer was washed with brine, dried over $\mathrm{Na}_{2} \mathrm{SO}_{4}$, and concentrated under vacuum. The residue was purified by flash column chromatography (silica gel, 12.5\% EtOAc in hexane) to give the product.

1,3-Diphenyl-prop-2-yn-1-ol: 93\% yield. 97\% ee detemined by HPLC analysis (Chiralcel OD column, IPA:hexane $=1: 10$ ). Retention time: $t_{\text {major }}=18.14 \mathrm{~min}$, $\mathrm{t}_{\text {minor }}=35.49 \min . \quad[\mathrm{a}]^{15}{ }_{\mathrm{D}}=+5 \quad\left(\mathrm{c}=0.89, \mathrm{CHCl}_{3}\right) .{ }^{1} \mathrm{H} \mathrm{NMR}\left(200 \mathrm{MHz}, \mathrm{CDCl}_{3}\right): \delta$ 7.60-7.32(m, 10H), 5.66(s, $1 \mathrm{H}), 2.39(\mathrm{br}, 1 \mathrm{H})$. Anal. Calcd. for $\mathrm{C}_{15} \mathrm{H}_{12} \mathrm{O}: \mathrm{C}, 86.54 ; \mathrm{H}$, 5.77. Found: C, 86.31; H, 5.93. (E.J.Corey, K.A.Cimprich. J. Am. Chem. Soc. 1994, 116, 3151.)

1-(4-Fluorophenyl)-3-phenyl-prop-2-yl-1-ol: $87 \%$ yield. $93 \%$ ee detemined by HPLC analysis (Chiralcel OD column, IPA:hexane $=1: 10$ ). Retention time: $\mathrm{t}_{\text {major }}=7.72$ $\min , \mathrm{t}_{\text {minor }}=29.46 \min .[\mathrm{a}]^{15} \mathrm{D}=+3\left(\mathrm{c}=0.94, \mathrm{CHCl}_{3}\right) .{ }^{1} \mathrm{H} \mathrm{NMR}\left(200 \mathrm{MHz}, \mathrm{CDCl}_{3}\right): \delta$ 7.58-7.45(m, 4H), 7.35-7.26(m, 3H), 7.12-6.97(m, 2H). 5.65(s, 1H), 2.41(br, $1 \mathrm{H})$. Anal. Calcd. for $\mathrm{C}_{15} \mathrm{H}_{11} \mathrm{OF}$ : C, 79.65; H, 4.87. Found: C, 79.77; H, 5.01. (David Moore, Lin Pu, Org. Lett. 2002, 4, 1855)

1-(2-Naphthyl)-3-phenyl-prop-2-yl-1-ol: 75\% yield. 98\% ee detemined by HPLC analysis (Chiralcel OD column, IPA:hexane $=1: 10$ ). Retention time: $t_{\text {major }}=16.51 \mathrm{~min}$, $\mathrm{t}_{\text {minor }}=59.38 \min .[\mathrm{a}]^{19} \mathrm{D}^{=}=-12\left(\mathrm{c}=2.42, \mathrm{CHCl}_{3}\right) .{ }^{1} \mathrm{H} \mathrm{NMR}\left(200 \mathrm{MHz}, \mathrm{CDCl}_{3}\right): \delta 7.92(\mathrm{~s}$, $1 \mathrm{H}), 7.73-7.68(\mathrm{~m}, 3 \mathrm{H}), 7.58(\mathrm{~s}, 1 \mathrm{H}) .7 .45-7.32(\mathrm{~m}, 4 \mathrm{H}), 7.26(\mathrm{~s}, 3 \mathrm{H}), 5.66(\mathrm{~s}, 1 \mathrm{H})$, 2.41(br, 1H). Anal. Calcd. for $\mathrm{C}_{19} \mathrm{H}_{14} \mathrm{O}$ : C, 88.37; H, 5.43. Found: C, 88.17; H, 5.41. (Li, Zhen; Upadhyay, Veena; DeCamp, Ann E.; DiMichele, Lisa; Reider, Paul J. Synthesis. 1999, 1453)

1-(1-Naphthyl)-3-phenyl-prop-2-yl-1-ol: 67\% yield. 91\% ee detemined by HPLC analysis (Chiralcel OD column, IPA:hexane $=1: 10$ ). Retention time: $t_{\text {major }}=16.92 \mathrm{~min}$, $\mathrm{t}_{\text {minor }}=39.61 \mathrm{~min} .[\mathrm{a}]^{19}{ }_{\mathrm{D}}=-26\left(\mathrm{c}=1.84, \mathrm{CHCl}_{3}\right) .{ }^{1} \mathrm{H} \mathrm{NMR}\left(200 \mathrm{MHz}, \mathrm{CDCl}_{3}\right): \delta 8.28(\mathrm{~d}$, $\mathrm{J}=7.6,1 \mathrm{H}), 7.78-7.66(\mathrm{~m}, 3 \mathrm{H}), 7.54-7.38(\mathrm{~m}, 5 \mathrm{H}) .7 .23-7.16(\mathrm{~m}, 3 \mathrm{H}), 6.23(\mathrm{~s}, 1 \mathrm{H})$, 2.24(br, 1H). Anal. Calcd. for $\mathrm{C}_{19} \mathrm{H}_{14} \mathrm{O}: \mathrm{C}, 88.37$; H, 5.43. Found: C, 88.24; H, 5.62. (David Moore, Lin Pu, Org. Lett. 2002, 4, 1855)

1-(2-Methoxyphenyl)-3-phenyl-prop-2-yl-1-ol: $89 \%$ yield. 97\% ee detemined by HPLC analysis (Chiralcel OD column, IPA:hexane=1:10). Retention time: $\mathrm{t}_{\text {major }}=16.28 \mathrm{~min}, \mathrm{t}_{\text {minor }}=22.23 \mathrm{~min} .[\mathrm{a}]^{18}{ }_{\mathrm{D}}=-8\left(\mathrm{c}=0.42, \mathrm{CHCl}_{3}\right) .{ }^{1} \mathrm{H} \mathrm{NMR}(200 \mathrm{MHz}$, $\left.\mathrm{CDCl}_{3}\right): \delta 7.67-7.63(\mathrm{~d}, \mathrm{~J}=7.4 \mathrm{~Hz}, 1 \mathrm{H}), \quad 7.47-7.45(\mathrm{~m}, 2 \mathrm{H}), \quad 7.32-7.25(\mathrm{~m}, 4 \mathrm{H})$, 7.00-6.91(m, 2H), 5.93(m, 1H). 3.93(s, 3H), 3.13(s, 1H). Anal. Calcd. for $\mathrm{C}_{16} \mathrm{H}_{14} \mathrm{O}_{2}: \mathrm{C}$, 
80.67; H, 5.88. Found: C, 80.38; H, 5.31. (Li, Zhen; Upadhyay, Veena; DeCamp, Ann E.; DiMichele, Lisa; Reider, Paul J. Synthesis. 1999, 1453)

1-(3-Methoxyphenyl)-3-phenyl-prop-2-yl-1-ol: $85 \%$ yield. $95 \%$ ee detemined by HPLC analysis (Chiralcel OD column, IPA:hexane=1:10). Retention time: $\mathrm{t}_{\text {major }}=18.03 \mathrm{~min}, \mathrm{t}_{\text {minor }}=31.52 \mathrm{~min} .[\mathrm{a}]^{15}{ }_{\mathrm{D}}=+7\left(\mathrm{c}=0.77, \mathrm{CHCl}_{3}\right) .{ }^{1} \mathrm{H} \mathrm{NMR}(200 \mathrm{MHz}$, $\left.\mathrm{CDCl}_{3}\right): \delta 7.47-7.45(\mathrm{~m}, 2 \mathrm{H}), 7.33-7.17(\mathrm{~m}, 6 \mathrm{H}), 6.9(\mathrm{~m}, 1 \mathrm{H}) .5 .63(\mathrm{~s}, 1 \mathrm{H}), 3.79(\mathrm{~s}, 3 \mathrm{H})$, 2.40(brs, 1H). Anal. Calcd. for $\mathrm{C}_{16} \mathrm{H}_{14} \mathrm{O}_{2}$ : C, 80.67; H, 5.88. Found: C, 80.80; H, 5.71. (David Moore, Lin Pu, Org. Lett. 2002, 4, 1855)

1-(4-Methoxyphenyl)-3-phenyl-prop-2-yl-1-ol: $90 \%$ yield. $92 \%$ ee detemined by HPLC analysis (Chiralcel OD column, IPA:hexane=1:10). Retention time: $\mathrm{t}_{\text {major }}=49.79 \mathrm{~min}, \mathrm{t}_{\text {minor }}=71.37 \mathrm{~min} .[\mathrm{a}]^{15} \mathrm{D}=+3\left(\mathrm{c}=0.93, \mathrm{CHCl}_{3}\right) .{ }^{1} \mathrm{H} \mathrm{NMR}(200 \mathrm{MHz}$, $\left.\mathrm{CDCl}_{3}\right): \delta 7.55-7.45(\mathrm{~m}, 2 \mathrm{H}), 7.32-7.23(\mathrm{~m}, 5 \mathrm{H}), 6.93-6.84(\mathrm{~m}, 2 \mathrm{H}) .5 .62(\mathrm{~s}, 1 \mathrm{H}), 3.86(\mathrm{~s}$, $3 \mathrm{H}), 2.31$ (brs, $1 \mathrm{H}) .{ }^{13} \mathrm{C} \mathrm{NMR}\left(50 \mathrm{MHz}, \mathrm{CDCl}_{3}\right): \delta 159.7,136.8,133.1,131.8,128.6$, 128.3, 127.2, 113.9, 89.2, 86.6, 65.9, 55.3. IR(KBr): 3369, 3062, 2875, 2836, 2188, 1248, 1034, 832, 758, $691 \mathrm{~cm}^{-1}$. HRMS(FAB), m/z : $\left[\mathrm{M}+1-\mathrm{H}_{2} \mathrm{O}\right]^{+}$, Calac: 221.0961. Found: 221.0959. Anal. Calcd. for $\mathrm{C}_{16} \mathrm{H}_{14} \mathrm{O}_{2}$ : C, 80.67; H, 5.88. Found: C, 80.80; H, 5.71 .

1-(3-Bromophenyl)-3-phenyl-prop-2-yl-1-ol: $91 \%$ yield. $94 \%$ ee detemined by HPLC analysis (Chiralcel OD column, IPA:hexane $=1: 10$ ). Retention time: $\mathrm{t}_{\text {major }}=11.26$ $\min , \mathrm{t}_{\text {minor }}=48.63 \mathrm{~min} .[\mathrm{a}]^{15} \mathrm{D}=+6\left(\mathrm{c}=0.92, \mathrm{CHCl}_{3}\right) .{ }^{1} \mathrm{H} \mathrm{NMR}\left(200 \mathrm{MHz}, \mathrm{CDCl}_{3}\right): \delta$ $7.76(\mathrm{~m}, 1 \mathrm{H}), 7.48-7.21(\mathrm{~m}, 8 \mathrm{H}), 5.62(\mathrm{~s}, 1 \mathrm{H}) .2 .57(\mathrm{brs}, 1 \mathrm{H}) .{ }^{13} \mathrm{C}$ NMR $(50 \mathrm{MHz}$, $\left.\mathrm{CDCl}_{3}\right): \delta 146.9,143.0,131.5,130.2,129.8,129.0,128.5125 .3,122.5,88.3,86.7$, 65.7. IR(KBr): 3348, 3060, 2875, 2230, 1592, 1487, 1070, 1010, 903, 822, 756, 691 $\mathrm{cm}^{-1}$. HRMS(FAB), m/z : $\left[\mathrm{M}+1-\mathrm{H}_{2} \mathrm{O}\right]^{+}$, Calac: 268.9960. Found: 268.9959. Anal. Calcd. for $\mathrm{C}_{15} \mathrm{H}_{11} \mathrm{OBr}$ : C, 62.74; H, 3.83. Found: C, 62.97; H, 3.71.

1-(4-Bromophenyl)-3-phenyl-prop-2-yl-1-ol: $85 \%$ yield. $92 \%$ ee detemined by HPLC analysis (Chiralcel OD column, IPA:hexane=1:10). Retention time: $\mathrm{t}_{\text {major }}=10.64 \mathrm{~min}, \mathrm{t}_{\text {minor }}=39.58 \mathrm{~min} .[\mathrm{a}]^{15} \mathrm{D}=+4\left(\mathrm{c}=0.76, \mathrm{CHCl}_{3}\right) .{ }^{1} \mathrm{H} \mathrm{NMR}(200 \mathrm{MHz}$, $\left.\mathrm{CDCl}_{3}\right): \delta 7.51-7.41(\mathrm{~m}, 4 \mathrm{H}), 7.35-7.29(\mathrm{~m}, 3 \mathrm{H}), 7.22-7.15(\mathrm{~m}, 2 \mathrm{H}), 5.61(\mathrm{~s}, 1 \mathrm{H})$. 2.94(brs, $1 \mathrm{H}) .{ }^{13} \mathrm{C} \mathrm{NMR}\left(50 \mathrm{MHz}, \mathrm{CDCl}_{3}\right): \delta 143.5,139.7,131.5,128.7,128.3,127.7$, 122.2 121.1, 88.3, 86.8, 65.8. IR(KBr): 3344, 3060, 2960, 2232, 1570, 1471, 1426 , 1071, 1041, 785, $695 \mathrm{~cm}^{-1}$. HRMS(FAB), m/z : $\left[\mathrm{M}+1-\mathrm{H}_{2} \mathrm{O}\right]^{+}$, Calac: 268.9960. Found: 268.9960. Anal. Calcd. for $\mathrm{C}_{15} \mathrm{H}_{11} \mathrm{OBr}$ : C, 62.74; H, 3.83. Found: C, 62.71; H, 4.03 .

4-Methyl-1-phenyl-pent-1-yl-3-ol: $88 \%$ yield. 75\% ee detemined by HPLC analysis (Chiralcel OD column, IPA:hexane $=1: 10$ ). Retention time: $t_{\text {major }}=6.13 \mathrm{~min}$, $\mathrm{t}_{\text {minor }}=10.68$ min. $[\mathrm{a}]^{15}{ }_{\mathrm{D}}=-2 \quad\left(\mathrm{c}=0.69, \mathrm{CHCl}_{3}\right) .{ }^{1} \mathrm{H} \quad \mathrm{NMR}\left(200 \mathrm{MHz}, \mathrm{CDCl}_{3}\right)$ : 87.46-7.41(m, 2H), 7.32-7.29(m, 3H), 4.39(d, J=5.6Hz, 1H). 2.26(brs, $1 \mathrm{H})$, 1.99-1.93(m,1H), 1.07(d, J=6.4Hz, 6H). Anal. Calcd. for $\mathrm{C}_{12} \mathrm{H}_{14} \mathrm{O}: \mathrm{C}, 82.76 ; \mathrm{H}, 8.05$. 
Found: C, 82.71; H, 8.03. (Matsumura, K.; Hashiguchi, S.; Ikariya, T.; Noyori, R. J. Am. Chem. Soc. 1997, 119, 8738.)

1-phenyl-hex-1-yn-3-ol: $82 \%$ yield. 85\% ee detemined by HPLC analysis (Chiralcel OD column, IPA:hexane $=1: 10)$. Retention time: $t_{\text {major }}=6.28 \mathrm{~min}, \mathrm{t}_{\text {minor }}=15.93 \mathrm{~min}$. $[\mathrm{a}]^{15}{ }_{\mathrm{D}}=+3 \quad\left(\mathrm{c}=0.75, \mathrm{CHCl}_{3}\right) .{ }^{1} \mathrm{H} \quad \mathrm{NMR}\left(200 \mathrm{MHz}, \mathrm{CDCl}_{3}\right): \delta 7.45-7.42(\mathrm{~m}, 2 \mathrm{H})$, 7.33-7.28(m, 3H), 4.61(t, J=6.2Hz, 1H). 2.26(brs, $1 \mathrm{H}), 1.85-1.74(\mathrm{~m}, 2 \mathrm{H}), 1.61-1.50(\mathrm{~m}$, $2 \mathrm{H}), 0.98(\mathrm{t}, \mathrm{J}=7.4 \mathrm{~Hz}, 3 \mathrm{H}) .{ }^{13} \mathrm{C} \mathrm{NMR}\left(50 \mathrm{MHz}, \mathrm{CDCl}_{3}\right): \delta 131.6,128.3,128.2,122.7$, 90.3, 84.7, 62.7, 39.9, 29.7, 13.7. IR(KBr): 3331, 3059, 2959, 2933, 2871, 2227, 1598, 1489, 1066, 1026, 756, $691 \mathrm{~cm}^{-1}$. HRMS(FAB), m/z: $\left[\mathrm{M}+1-\mathrm{H}_{2} \mathrm{O}\right]^{+}$, Calac: 157.1012. Found: 157.1012. Anal. Calcd. for $\mathrm{C}_{12} \mathrm{H}_{14} \mathrm{O}$ : C, 82.76; H, 8.05. Found: C, 82.84; H, 7.71 .

1-(4-Chlorophenyl)-3-phenyl-prop-2-yl-1-ol: $81 \%$ yield. $92 \%$ ee detemined by HPLC analysis (Chiralcel OD column, IPA:hexane=1:10). Retention time: $\mathrm{t}_{\text {major }}=10.15 \mathrm{~min}, \mathrm{t}_{\text {minor }}=36.96 \mathrm{~min} .[\mathrm{a}]^{15} \mathrm{D}=+6\left(\mathrm{c}=0.72, \mathrm{CHCl}_{3}\right) .{ }^{1} \mathrm{H} \mathrm{NMR}(200 \mathrm{MHz}$, $\left.\mathrm{CDCl}_{3}\right): \delta 7.54-7.45(\mathrm{~m}, 4 \mathrm{H}), 7.33-7.25(\mathrm{~m}, 5 \mathrm{H}), 5.63(\mathrm{~s}, 1 \mathrm{H}) .2 .30(\mathrm{brs}, 1 \mathrm{H})$. Anal. Calcd. for $\mathrm{C}_{15} \mathrm{H}_{11} \mathrm{OCl}$ : C, 74.24; H, 4.54. Found: C, 74.51; H, 4.33. (David Moore, Lin Pu, Org. Lett. 2002, 4, 1855).

1,5-Diphenyl-pent-1-en-4-yl-3-ol: 71\% yield. 93\% ee detemined by HPLC analysis (Chiralcel OD column, IPA:hexane $=1: 10$ ). Retention time: $t_{\text {major }}=22.58 \mathrm{~min}$, $\mathrm{t}_{\text {minor }}=71.62 \min . \quad[\mathrm{a}]^{18}{ }_{\mathrm{D}}=+1 \quad\left(\mathrm{c}=2.58, \mathrm{CHCl}_{3}\right) .{ }^{1} \mathrm{H} \quad \mathrm{NMR}\left(200 \mathrm{MHz}, \mathrm{CDCl}_{3}\right)$ : 67.47-7.23(m, 10H), 6.88-6.80(d, J=15.8Hz, 1H). 6.33-6.26(m, $1 \mathrm{H}), 5.28(\mathrm{~d}, \mathrm{~J}=5.6 \mathrm{~Hz}$, 1H), 2.04(s, 1H). (Gao, G.; Moore, D.; Xie, R. G.; Pu, L. Org. Lett. 2002, 4, 4143) 